\title{
Les Altérations Hydrothermales Associées À La Minéralisation Aurifère Du Gisement De Dougbafla (District d'Oumé-Hiré, Centre-Ouest De La Côte d'Ivoire)
}

\author{
Ouattara Aboubakar Sidik \\ Coulibaly Yacouba \\ Kouadio Fossou J-L. H.
}

Laboratoire de Géologie du Socle et de Métallogénie, UFR des Sciences de la Terre et des Ressources Minières, Université Félix HOUPHOÜT-BOIGNY, Côte d'Ivoire.

Doi: 10.19044/esj.2017.v13n30p108 URL:http://dx.doi.org/10.19044/esj.2017.v13n30p108

\begin{abstract}
The Dougbafla gold deposit is located in the West-Central part of Côte d'Ivoire at about $240 \mathrm{~km}$ from Abidjan, on the Birimian greenstone belt of Fettèkro (West African craton). The lithologies of this deposit can be divided into three lithotectonic units which correspond to volcanic, sedimentary, and plutonic assemblages metamorphosed in the shale facies. Hydrothermalism, on the one hand, caused a pervasive alteration of the primary paragenesis marked by sericitic, silica, and carbonate alteration. On the other hand, it causes a vein alteration materialized by quartz veins. These hydrothermal alterations induced two types of gold mineralization in the Dougbafla deposit. These are: (i) disseminated gold and sulphide mineralization in the granophyre associated with sericite, silica and dolomite alteration in which no quartz vein has been reported; this type however is controlled by the intrusion of granophyre and (ii) a quartz vein mineralization controlled by deformation.
\end{abstract}

Keywords: Dougbafla, Birimian, Hydrothermalism, sericitic, silica

\section{Résumé}

Le gisement aurifère de Dougbafla est situé à $240 \mathrm{~km}$ d'Abidjan au Centre-Ouest de la Côte d'Ivoire sur la ceinture de roches vertes birimiennes de Fettèkro (craton ouest Africain). Les lithologies de ce gisement peuvent être subdivisées en trois unités lithotectoniques correspondant à des assemblages volcaniques, sédimentaires et plutoniques métamorphisées dans 
le faciès des schistes verts. L'hydrothermalisme a provoqué d'une part, une altération pervasive de la paragenèse primaire des marquée par la séricitisation, la silicification et la carbonisation et d'autre part par une altération filonienne matérialisée par des veines de quartz. Ces altérations hydrothermales ont induit deux types de minéralisation aurifère dans le gisement de Dougbafla. Il s'agit : (i) d'une minéralisation disséminée à or et sulfures dans le granophyre altéré en séricite, en silice et en dolomite dans lequel aucun filon de quartz n'a été signalé; ce type est contrôlé par l'intrusion de granophyre et (ii) d'une minéralisation filonienne liée à des veines de quartz, contrôlée par la déformation.

Mots-clés: Dougbafla, Birimien, Hydrothermalisme, séricitisation, silicification

\section{Introduction}

L'exploitation de l'or dans la région d'Oumé située au centre-ouest de la Côte d'Ivoire a commencé il y'a plusieurs siècles, sous forme artisanale par les populations locales. De nombreux vestiges (fosses et des puits) artisanaux qui coïncident généralement avec les anomalies géochimique sol en or l'atteste (Sonnendrucker, 1969). L'exploitation des filons de quartz se poursuit toujours de nos jours. Les travaux modernes menés ont permis de mettre en évidence dans la zone Dougbafla-Bandama un gisement dénommé Dougbafla d'environ 14 tonnes d'or pour une teneur moyenne de $2 \mathrm{~g} / \mathrm{t}$ exploité en 2014 par la compagnie Newcrest mining Ltd. L'hydrothermalisme a provoqué dans les roches encaissantes un certain nombre d'altérations étroitement associées à la minéralisation aurifère ce gisement.

L'objectif visé par cette étude est la caractérisation de ces altérations, qui permettra une meilleure connaissance des différents types de minéralisation aurifère dans le secteur minier de Dougbafla-Bandama.

\section{Contexte Geologique}

La région d'Oumé dans laquelle s'inscrit le gisement de Dougbafla, est située au sud de la ceinture de roches vertes d'Oumé-Fettékro, à $45 \mathrm{~km}$ au nord de Divo et à environ $240 \mathrm{~km}$ au nord-ouest de la ville d'Abidjan. Cette ceinture appartenant au domaine d'âge protérozoïque à la dorsale de Man (Figure 1 et 2 ) est constituée d'un assemblage de roches birimiennes volcaniques et sédimentaires métamorphisé dans le faciès des schistes verts (Yacé, 1984). Des granitoïdes post-tectoniques font intrusion dans les lithologies de cette ceinture (Yacé, 1984; Mortimer, 1992; Daouda, 1998).

Les formations birimiennes ouest-africaine ont été structuré au cours du cycle orogénique éburnéen daté entre 2,5 et 1,6 Ga. (Arnould, 1945; 
Roques, 1948; Soule De Lafont, 1956; Arnould, 1961; Bonhomme, 1962; Marcelin, 1971; Tagini, 1971; Bard, 1974; Yacé, 1984; Doumbia et al., 1998; Feybesse et al., 1989; Milési et al., 1989; Abouchami, 1990; et Boher et al., 1992).

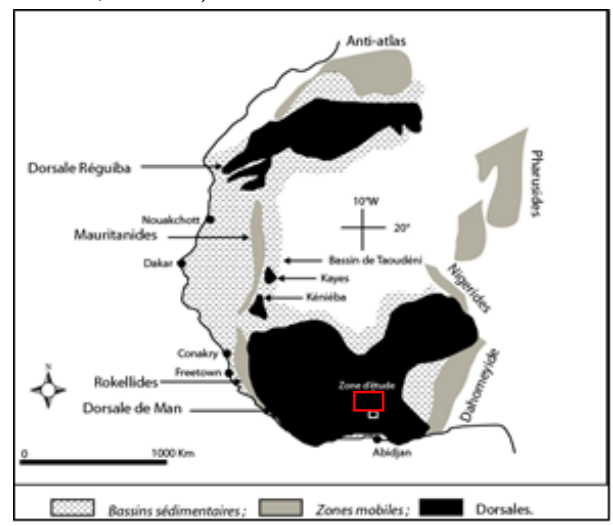

Figure 1: Le craton ouest africain d'après Tempier (1986, modifié).

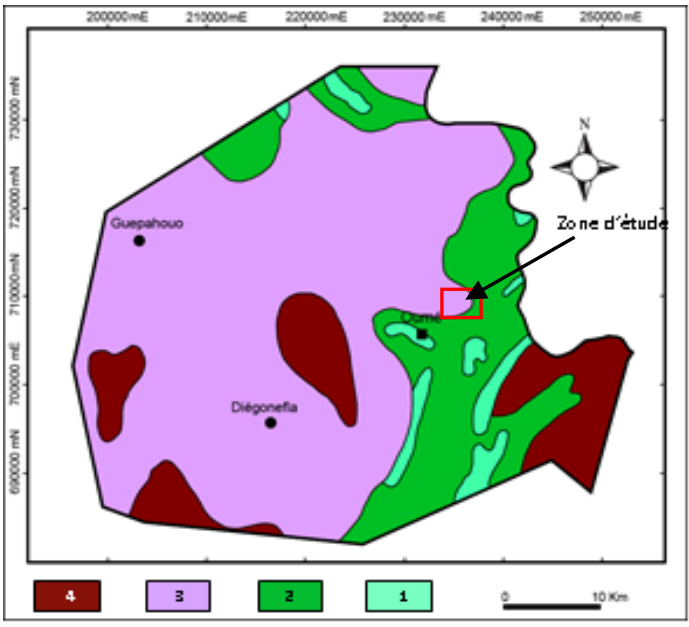

Figure 2 : Carte géologique de la région d'Oumé (Géomines, 1982, modifiée).

1) Métavocanite ; 2) Métasédiment ; 3) Granites hétérogènes ; 4) Granitoïdes intrusifs

Concernant la minéralisation en or, le sillon birimien d'OuméFettékro abrite le plus grand nombre de gisements actuellement en exploitation en Côte d'Ivoire : (i) le gisement de Bonikro (37 tonnes d'or), (ii) le gisement d'Agbaou (23 tonnes d'or), (iii) le gisement de Dougbafla (14 tonnes d'or) et (iv) le gisement d'Hiré (20 tonnes d'or). Ces quatre gisements d'or sont liés à des lentilles de quartz et à des sulfures disséminés développés dans différentes lithologies fortement hydrothermalisées telles que les volcanites mafiques à Agbahou (Houssou, 2013), les granodiorites à Bonikro (Ouattara, 2015) et Hiré (Sonnendrucker, 1969) et dans les granophyres à Dougbafla.

\section{Methodologie}

Les différents intervalles minéralisés et les minéraux métalliques ainsi que des grains d'or ont été décrits à l'œil nu et à l'aide d'une loupe binoculaire Zeiss. L'étude des lames minces polies réalisée au microscope métallographique du type Nachet, au microscope électronique à balayage (MEB) au Centre d'Analyses et de Recherche (CAR) de la PETROCI a permis d'identifier les paragenèses métallifères liées à l'or.

L'analyse ponctuelle des phases cristallines sur treize lames minces polies et métallisées au carbone, a été réalisée à la microsonde électronique CAMECA SX100 au service commun des microscopies électroniques et 
microanalyses (SCMEM) du laboratoire de Géoressources de l'université de Lorraine, Nancy en France. L'analyse a été faite sous une tension de $15 \mathrm{kV}$, permettant de produire un faisceau de courant de $12 \mathrm{nA}$ et $20 \mathrm{nA}$ pour les sulfures avec un temps d'intégration de $4 \mathrm{~s}$.

L'analyse chimique, en vue de déterminer la teneur en or des différents échantillons a été réalisée par spectrométrie d'absorption atomique au laboratoire d'analyse S.G.S sis au Ghana et au laboratoire ALS minerals au Canada.

\section{Resultats}

\section{- $\quad$ Pétrographie des roches encaissantes}

La minéralisation aurifère de Dougbafla-Bandama a été observée dans la plupart des formations volcaniques (basaltes, andésites et rhyodacites), volcanoclastites (brèches et Hyaloclastites), sédimentaires (schistes noirs, schistes sériciteux et schistes chloriteux) et dans les intrusifs (granites, granophyres, granodiorites, microgranodiorites, tonalites, microdiorites, épidiorites et lamprophyres). Néanmoins, même si toutes ces lithologies peuvent encaisser la minéralisation, une différence subsiste. En effet, les granophyres apparaissent presque toujours minéralisés. Les granophyres sont des roches massives, leucocrates à grains moyens donnant l'aspect de microgranites. Ils ont une minéralogie constituée essentiellement de phénocristaux de plagioclases subautomorphe en partie envahis par de fines plages de séricite, d'albite, de muscovite, de biotite et de quartz (Figure 3). On note accessoirement la présence d'orthoses, d'épidote, de magnétites /hématites, et de sulfures.
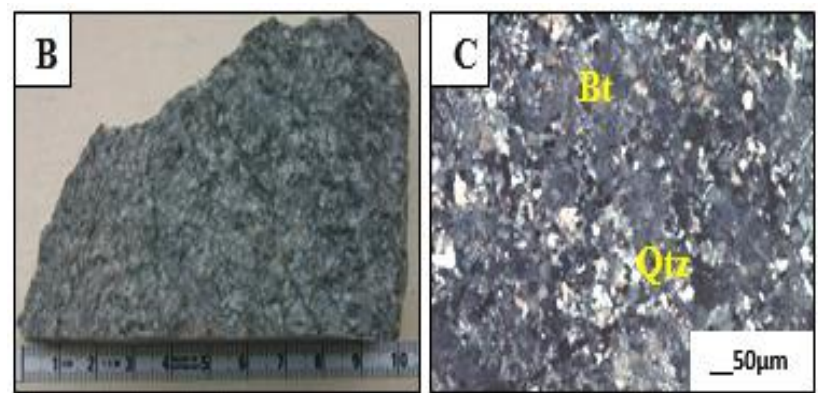

Figure 3 : Aspects macroscopique et microscopique du granophyre.

A : Echantillon d'affleurement ; B : Microphotographie. $B t=$ Biotite $;$ Plag $=$ Plagioclase $;$ Qtz $=$ Quartz

\section{- Altération hydrothermale}

L'altération hydrothermale est omniprésente dans les différentes unités lithologiques de la zone d'étude. Leurs distributions sont gouvernées par: (i) le mode pervasif ou diffus, souvent d'extension régionale, portant sur pseumorphose des principaux minéraux primaires de l'encaissant et (ii) le 
mode filonien, d'extension locale, généralement contrôlée par une porosité de fracture.

\section{- $\quad$ Altération pervasive}

Cette altération affecte presque toutes les lithologies de la zone d'étude. La carbonatation (calcite et/ou dolomite), la chloritisation et la séricitisation sont les altérations pervasives les plus importants de la zone de Dougbafla-Bandama. A un degré moindre, on observe la silicification, l'épidotisation, l'hématisation et l'albitisation.

\section{Carbonatation}

Cette altération est principalement observée dans les basaltes, les granophyres, les microgranodiorites, les andésites, les microdiorites, les volcanoclastites et les schistes chloriteux. La carbonatation résulte de l'altération des plagioclases et feldspaths alcalins. La calcite est couramment rencontrée et à un degré moindre, les dolomites (Figure. 4). Dans le diagramme Fe-Ca-Mg et dans celui de Trdlicka et Hoffman (1976), les compositions chimiques des carbonates analysés (Tableau I), tombent respectivement dans les champs des calcites et dans les champs des dolomites ferrifères, ou quelque fois dans celui des dolomites (Figure 5).
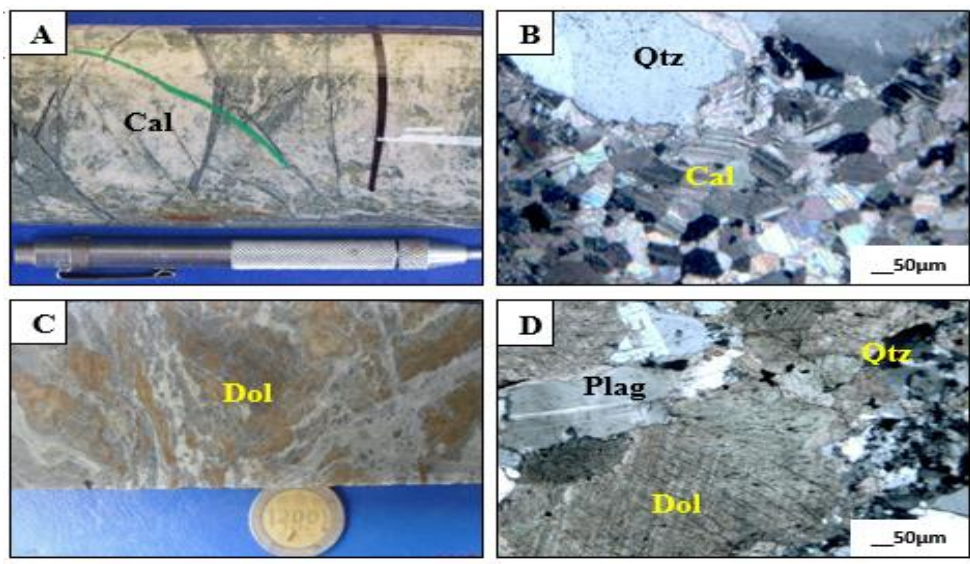

Figure 4 : Aspects macroscopiques et microscopiques de la carbonatation.

A : Carotte de microdiorite fortement altéré en calcite; $\mathbf{B}:$ Microphotographie de la microdiorite altéré en calcite; $\mathbf{C}$ : Carotte de granophyre intensément altérée en dolomite; D : Microphotographie du granophyre altéré en dolomite

Cal $=$ calcite $;$ Dol $=$ dolomite $;$ Plag $=$ Plagioclase $; Q t z=Q u a r t z$

Tableau I : Composition chimique des carbonates observés à Dougbafla Est.

\begin{tabular}{ccccccc}
\hline $\mathrm{N}^{\circ} \mathrm{Ech}$ & $\begin{array}{c}\text { OADE112- } \\
187,7 / \mathrm{IF}\end{array}$ & $\begin{array}{c}\text { OADE113- } \\
\text { 253,3/BAS }\end{array}$ & $\begin{array}{c}\text { OADE114- } \\
253,3 / \mathrm{AND}\end{array}$ & $\begin{array}{c}\text { OADE115- } \\
258,9 / \mathrm{SCH}\end{array}$ & $\begin{array}{c}\text { DEDD001- } \\
160,7 / \mathrm{SCH}\end{array}$ & $\begin{array}{c}\text { HDD2063c- } \\
166,9 / \mathrm{BAS}\end{array}$ \\
\hline $\mathrm{CaO}$ & 29,84 & 28,72 & 29,4 & 30,99 & 52,63 & 54,33 \\
$\mathrm{MgO}$ & 13,1 & 13,88 & 13,26 & 17,5 & 0,58 & 0,00 \\
$\mathrm{FeO}$ & 11,87 & 10,24 & 11,16 & 6,32 & 1,20 & 0,00 \\
$\mathrm{MnO}$ & 0,22 & 0,00 & 0,00 & 0,28 & 0,27 & 0,00 \\
$\mathrm{SrO}$ & 0,00 & 0,00 & 0,00 & 0,00 & 0,00 & 0,00
\end{tabular}




\begin{tabular}{ccccccc} 
Total & 55,03 & 52,84 & 53,82 & 55,09 & 54,68 & 54,33 \\
\multicolumn{2}{l}{ Formule structurale } & $(23$ oxygènes $)$ & & & & \\
$\mathrm{Ca}$ & 11,935 & 11,790 & 11,955 & 11,783 & 26,995 & 28,00 \\
$\mathrm{Mg}$ & 7,290 & 7,928 & 7,503 & 9,258 & 0,417 & 0,00 \\
$\mathrm{Fe} 2+$ & 3,706 & 3,281 & 3,542 & 1,876 & 0,479 & 0,00 \\
$\mathrm{Mn}$ & 0,070 & 0,000 & 0,000 & 0,084 & 0,109 & 0,00 \\
$\mathrm{OH}^{*}$ & 2,000 & 2,000 & 2,000 & 2,000 & 2,000 & 0,00 \\
Total & 25,001 & 24,999 & 25,000 & 25,001 & 30,00 & 30,00 \\
\hline
\end{tabular}

$\mathbf{I F}=$ granophyre $; \mathbf{B A S}=$ Basalte $; \mathbf{A N D}=$ Andésite $; \mathbf{S C H}=$ Schiste noir

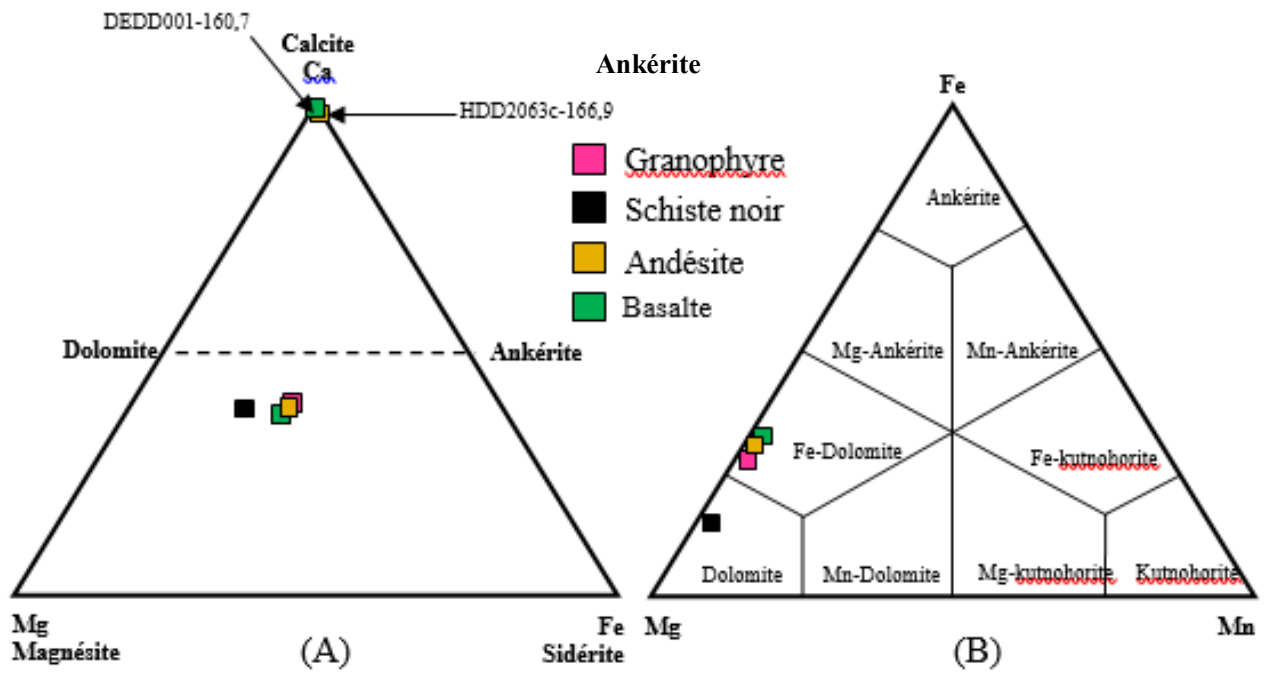

Figure 5 : (A) Calcites de Dougbafla dans le diagramme Mn-Ca-Mg; (B) Dolomites

\section{Chloritisation} dans le diagramme de Trdlicka et Hoffman (1976).

La chloritisation induite principalement par le métamorphisme épizonal (faciès schiste vert) se manifeste surtout au niveau des cristaux de biotite abondant dans les plutonites felsiques (granophyres et rhyodacite) mais également sur les phénocristaux de hornblende brune des métavolcanites (basalte et andésite) (Figure 6). Les chlorites sont les principaux marqueurs des zones non minéralisées à l'œil nu. D'après le diagramme de Bayliss (1975) les granophyres, les andésites et les sédiments contiennent principalement des chlorites de type ripidolite (Fig. 7). On note accessoirement des chlorites de type pycnochlorite dans les granophyres. La gamme de températures de cristallisation des chlorites selon le géothermomètre "chlorite" de Cathelineau et Nieva (1985) dans les schistes noirs et les andésites varie entre 315 et $328^{\circ} \mathrm{C}$, tandis que celle des granophyres, est legèrement moins chaude, variant entre 298 et $304^{\circ} \mathrm{C}$ (TableauVII). 
Tableau II : Compositions chimiques des chlorites observées à Dougbafla.

\begin{tabular}{|c|c|c|c|c|c|c|}
\hline $\mathbf{N}^{\circ} \mathbf{E c h}$ & 1/SO & 2/SO & 3/AN & 4/AN & $5 / \mathrm{FI}$ & 6/FI \\
\hline $\mathrm{SiO} 2$ & 24,82 & 24,02 & 25,59 & 24,64 & 27,56 & 29,39 \\
\hline $\mathrm{Al} 2 \mathrm{O} 3$ & 23,93 & 22,21 & 22,55 & 24,09 & 23,75 & 23,45 \\
\hline $\mathrm{MgO}$ & 15,06 & 14,32 & 11 & 11,69 & 20,67 & 19,72 \\
\hline $\mathrm{FeO}$ & 23,41 & 23,17 & 28,06 & 28,7 & 16,23 & 16,31 \\
\hline $\mathrm{MnO}$ & 0,84 & 0,84 & 0,85 & 0,85 & 0,84 & 0,84 \\
\hline $\mathrm{Cr} 2 \mathrm{O} 3$ & 0 & 0 & 0 & 0 & 0,41 & 0,38 \\
\hline Total & 87,22 & 83,72 & 87,2 & 89,12 & 88,62 & 89,25 \\
\hline \multicolumn{7}{|c|}{ Formule structurale } \\
\hline $\mathbf{S i}$ & 5,186 & 5,253 & 5,474 & 5,175 & 5,435 & 5,725 \\
\hline AlIV & 5,896 & 5,725 & 5,684 & 5,965 & 5,521 & 5,386 \\
\hline $\mathrm{Mg}$ & 4,692 & 4,667 & 3,507 & 3,661 & 6,076 & 5,726 \\
\hline $\mathrm{Fe}$ & 4,092 & 4,238 & 5,019 & 5,041 & 2,676 & 5,726 \\
\hline $\mathrm{Cr} 2 \mathrm{O} 3$ & 0 & 0 & 0 & 0 & 0,064 & 0,059 \\
\hline Total & 19,866 & 19,883 & 19,684 & 19,842 & 19,772 & 22,622 \\
\hline $\mathrm{XFe}$ & 0,47 & 0,48 & 0,59 & $\mathbf{0 , 5 8}$ & $\mathbf{0 , 3 1}$ & $\mathbf{0 , 5 0}$ \\
\hline $\mathbf{T}^{\circ} \mathrm{C}$ & 324,563 & 315,542 & 313,236 & 328,191 & 304,676 & 298,466 \\
\hline
\end{tabular}
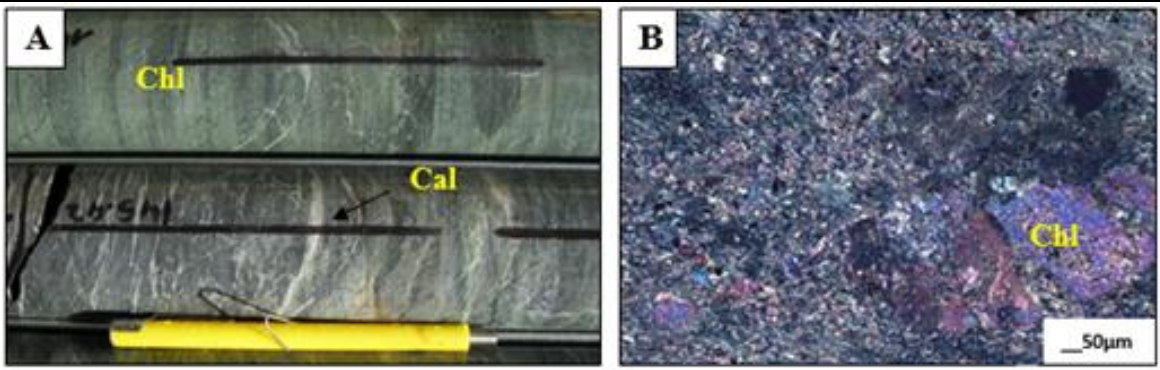

Figure 6. Aspects macroscopique et microscopique de la chloritisation

A : Carotte de basalte chloritisée de couleur verdâtre contrastant avec une autre non chloritisée (en gris noirâtre); B : Microphotographie montrant la chloritisation (en bleu violacé).

$$
\text { Chl }=\text { Chlorite } \text { Cal }=\text { calcite }
$$
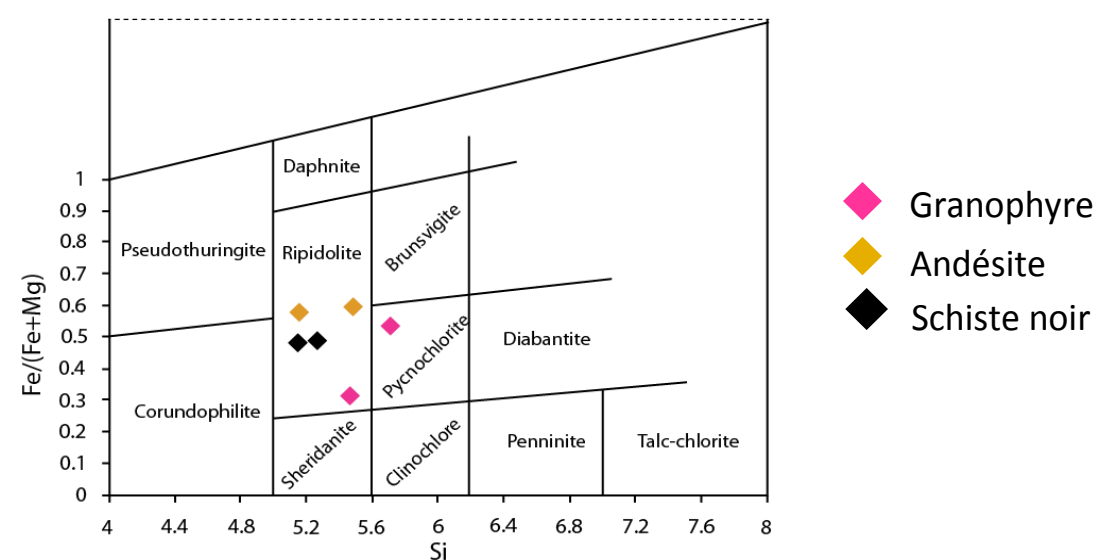

Figure 7 : Diagramme de composition des chlorites de Bayliss (1975) appliqué aux chlorites du Séricitisation gisement de Dougbafla.

La séricitisation résultant d'un remplacement partiel ou total des feldspaths et de la biotite est l'altération pervasive la plus présente dans la zone d'étude. Elle est généralement de forte intensité dans les felsites 
(granophyre et rhyodacite). La séricitisation est un bon traceur des zones minéralisées.

$\mathrm{Au}$ microscope, elle se présente sous forme de petites paillettes de teinte jaunâtre, bleuâtre ou rougeâtre disséminées dans la matrice cryptocristalline des roches (Figure 8). L'analyse des séricites à la microsonde (Tableau III), des granophyres et des basaltes indique une composition de muscovite (Figure 9).

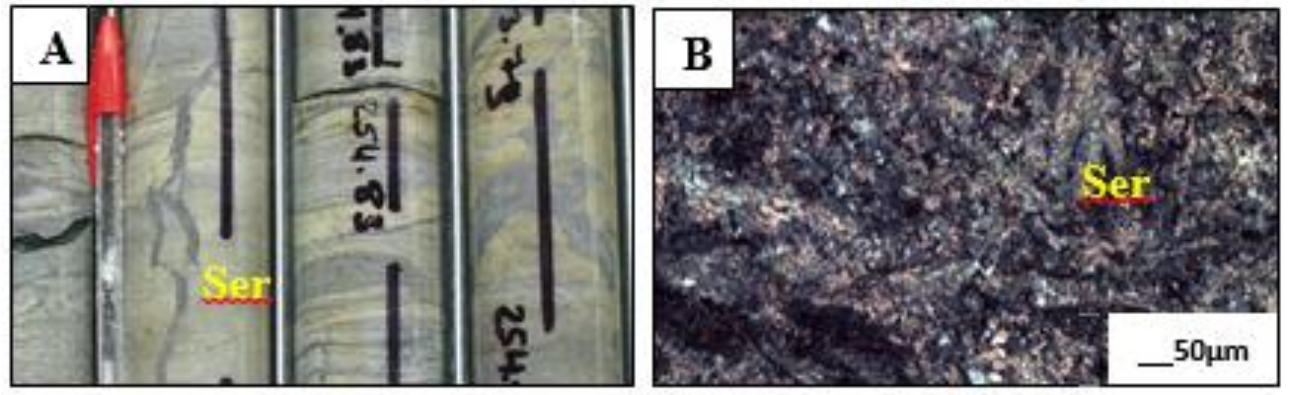

Figure 8: Aspects macroscopique et microscopique de la séricitisation.

A : Carotte de granophyre altérée en séricite ; B : Microphotographie.

Ser $=$ Séricite

\begin{tabular}{|c|c|c|c|c|c|c|c|c|c|c|}
\hline $\mathbf{N}^{\circ}$ Ech & IF16 & IF17 & IF18 & IF19 & IF20 & BAS4 & BAS7 & BAS8 & BAS9 & BAS10 \\
\hline $\mathrm{FeO}$ & 2,72 & 2,32 & 2,29 & 2,55 & 2,77 & 3,05 & 3,11 & 3,45 & 3,56 & 3,45 \\
\hline $\mathrm{Na} 2 \mathrm{O}$ & 0,00 & 0,11 & 0,02 & 0,07 & 0,15 & 0,00 & 0,00 & 0,00 & 0,00 & 0,00 \\
\hline $\mathrm{K} 2 \mathrm{O}$ & 11,33 & 10,46 & 9,89 & 10,78 & 11,10 & 12,26 & 11,87 & 12,02 & 11,99 & 11,55 \\
\hline $\mathrm{SiO} 2$ & 46,39 & 47,82 & 46,18 & 47,06 & 46,78 & 44,76 & 45,53 & 44,57 & 41,36 & 44,68 \\
\hline $\mathrm{MnO}$ & 0,03 & 0,00 & 0,02 & 0,00 & 0,00 & 0,04 & 0,04 & 0,00 & 0,06 & 0,01 \\
\hline $\mathrm{CaO}$ & 0,01 & 0,00 & 0,07 & 0,06 & 0,00 & 0,04 & 0,15 & 0,04 & 0,05 & 0,00 \\
\hline $\mathrm{A} 12 \mathrm{O} 3$ & 39,78 & 39,90 & 39,01 & 39,50 & 38,99 & 39,55 & 38,79 & 38,94 & 39,11 & 39,59 \\
\hline $\mathrm{TiO} 2$ & 0,78 & 0,54 & 0,01 & 0,01 & 0,00 & 0,01 & 0,00 & 0,00 & 0,09 & 0,07 \\
\hline $\mathrm{MgO}$ & 1,06 & 0,33 & 0,75 & 0,00 & 0,01 & 0,03 & 0,00 & 0,01 & 0,01 & 0,03 \\
\hline $\mathrm{Fe} 2 \mathrm{O} 3$ & 3,03 & 2,58 & 2,55 & 2,84 & 3,08 & 3,40 & 3,46 & 3,84 & 3,96 & 3,84 \\
\hline \multicolumn{11}{|c|}{ Formule structurale (22 oxygènes) } \\
\hline $\mathrm{Si}$ & 5,78 & 5,94 & 5,91 & 5,94 & 5,94 & 5,75 & 5,85 & 5,77 & 5,54 & 5,74 \\
\hline $\mathrm{Al}$ & 5,84 & 5,84 & 5,89 & 5,88 & 5,84 & 5,99 & 5,87 & 5,94 & 6,18 & 6,00 \\
\hline $\mathrm{Fe}$ & 0,28 & 0,24 & 0,25 & 0,27 & 0,29 & 0,33 & 0,33 & 0,37 & 0,40 & 0,37 \\
\hline $\mathrm{Mg}$ & 0,20 & 0,06 & 0,14 & 0,00 & 0,00 & 0,01 & 0,00 & 0,00 & 0,00 & 0,01 \\
\hline $\mathrm{Mn}$ & 0,00 & 0,00 & 0,00 & 0,00 & 0,00 & 0,00 & 0,00 & 0,00 & 0,00 & 0,00 \\
\hline $\mathrm{Ca}$ & 0,00 & 0,00 & 0,00 & 0,00 & 0,00 & 0,00 & 0,02 & 0,00 & 0,00 & 0,00 \\
\hline $\mathrm{Na}$ & 0,00 & 0,03 & 0,00 & 0,02 & 0,04 & 0,00 & 0,00 & 0,00 & 0,00 & 0,00 \\
\hline $\mathrm{K}$ & 1,80 & 1,66 & 1,62 & 1,74 & 1,80 & 2,01 & 1,94 & 1,98 & 2,05 & 1,89 \\
\hline $\mathrm{Ti}$ & 0,07 & 0,05 & 0,00 & 0,00 & 0,00 & 0,00 & 0,00 & 0,00 & 0,01 & 0,01 \\
\hline $\mathrm{Al}(\mathrm{IV})$ & 2,22 & 2,06 & 2,09 & 2,06 & 2,06 & 2,25 & 2,15 & 2,23 & 2,46 & 2,26 \\
\hline $\mathrm{Al}(\mathrm{VI})$ & 3,62 & 3,78 & 3,80 & 3,82 & 3,78 & 3,74 & 3,72 & 3,70 & 3,71 & 3,74 \\
\hline $\mathrm{Si}$ & 5,78 & 5,94 & 5,91 & 5,94 & 5,94 & 5,75 & 5,85 & 5,77 & 5,54 & 5,74 \\
\hline $\mathbf{Y}=\mathbf{T i}+\mathbf{A l}(\mathbf{V I})+\mathrm{Mn}+\mathrm{Mg}$ & 4,18 & 4,13 & 4,20 & 4,10 & 4,08 & 4,08 & 4,06 & 4,08 & 4,13 & 4,12 \\
\hline 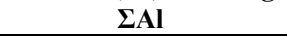 & 5,84 & 5,84 & 5,89 & $\mathbf{5 , 8 8}$ & 5,84 & 5,99 & $\mathbf{5 , 8 7}$ & 5,93 & 6,17 & 6,00 \\
\hline
\end{tabular}

Tableau III : Composition chimique des séricites observées à Dougbafla. 


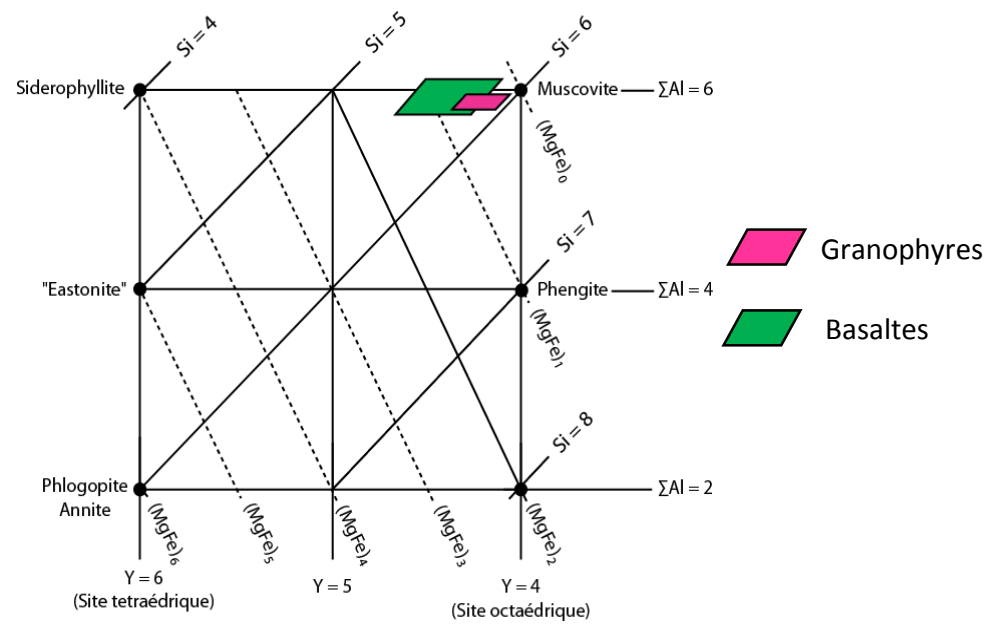

Figure 9 : Position des séricites de Dougbafla dans le digramme de Deer et al. (1992).

- $\quad$ Altération filonienne

L'altération filonienne se développe à la faveur de déformations cassante et cisaillante. Ce type d'altération est principalement matérialisé par les veines de quartz. Dans la zone d'étude, on observe trois types de veines qui sont: (i) les veines planaires; (ii) les veines irrégulières; et (iii) les veines en feuillet.

\section{Veines planaires}

Les veines planaires sont les plus répandues dans la zone d'étude.

Elles s'observent dans presque tous les faciès de la zone d'étude. Les veines planaires d'épaisseur pluricentimétriques sont principalement constituées de quartz laiteux ou enfumé souvent associé à la calcite (Figure 10A). Au microscope, la veine est à $95 \%$ constituée de quartz, de carbonate et de plagioclases (Figure 10B) avec quelques minéraux opaques de sulfures.

\section{Veines irrégulières}

Ce sont des veines laiteuses ou enfumées non planaires, sans aucune forme géométrique particulière. Ces veines de composition quartzofeldspathique (Figure 10C et 10D) s'observent généralement dans les zones de déformations ductiles.

\section{Veines en feuillet}

Ce sont des veinules blanchâtres millimétriques subparallèles entre elles et régulières composées de quartz $(70 \%)$, d'albite $(10 \%)$ et de calcite $(5 \%)$ (Figure 10E et 10F). Ces veines pouvant être confondu à la schistosité s'observent plus préférentiellement dans les granophyres et quelques rares fois, dans les basaltes. 

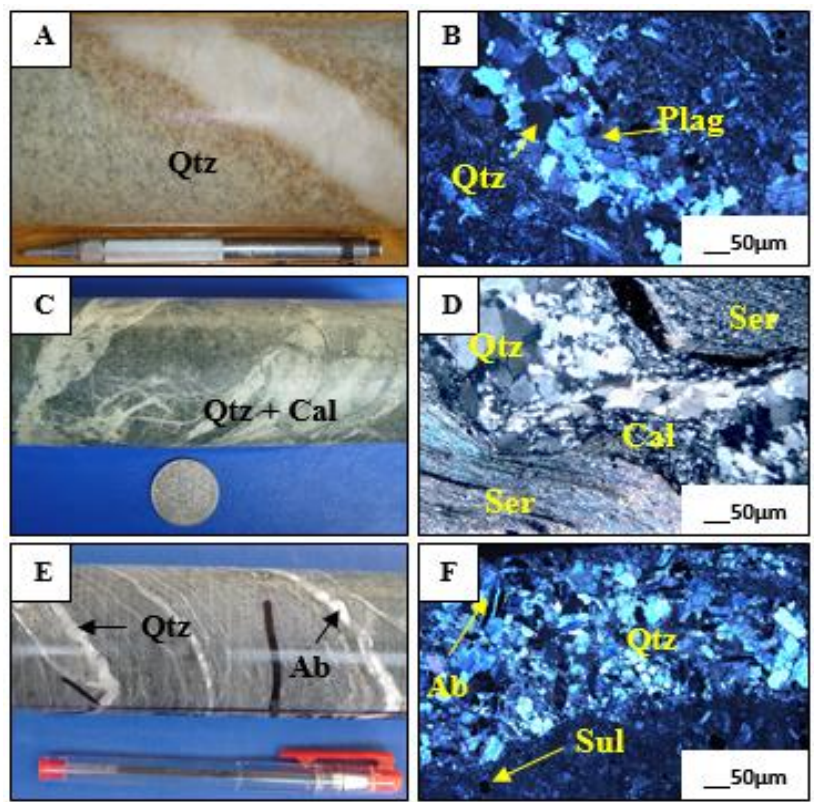

Figure 10 : Photographies et microphotographies 1llustrant des veines de Dougbafla. A : Photographie illustrant une veine de quartz planaire dans une carotte granophyre silicifié; B : Microphotographie d'une veine planaire de quartz; C : Aperçu macroscopique d'une veine irrégulière; D : Microphotographie de la veine irrégulière; E : Photographie de veines en feuillet à remplissage quartzo-albitique dans une rhyodacite; $\mathbf{F}$ : Microphotographie de la veine en feuillet dans la rhyodacite.

$Q t z=$ Quartz $;$ Cal $=$ Calcite $;$ Plag $=$ Plagioclase $; A b=$ Albite $;$ Ser $=$ Séricite $;$ Sul $=$ Sulfure

\section{- Minéralisation}

L'analyse des intervalles minéralisés de la zone d'étude, indique que l'or s'est mis en place selon deux modes qui sont : (i) le mode disséminé et (ii) le mode filonien.

\section{- Minéralisation disséminée}

Ce type de minéralisation encaissée dans presque toutes les lithologies de la zone d'étude est caractérisé par la dissémination de l'or et des sulfures dans l'encaissant. Néanmoins, elle s'observe majoritairement dans les intrusifs felsiques, notamment dans les granophyres associés à une moyenne à forte séricitisation et/ou silicification. Dans les basaltes apparaissent également de rares zones minéralisées sans altération hydrothermale (sans veines de quartz) ; cela est dû au fait que l'or primaire est inclus dans la matrice cryptocristalline des basaltes.

Un exemple de ce type de minéralisation, est attesté par l'intervalle minéralisé à $2,1 \mathrm{~g} / \mathrm{t}$ ) s'étendant de 60 à $100 \mathrm{~m}$ de profondeur (Figure 11) encaissé dans l'intrusion de granophyre intercepté par le forage FDC0831 réalisé à l'est de la zone d'étude. Dans cet intervalle minéralisé, le 
granophyre ne présente pas de déformation, ni de veines de quartz. Les teneurs en or les plus élevées, atteignant $4,96 \mathrm{~g} / \mathrm{t}$ entre 60 et $95 \mathrm{~m}$ de profondeur, ont un lien étroit avec la présence de sulfures disséminés, de séricite et de silice. A contrario, dans le granophyre sain (sans altération) intercepté entre 114 et $129 \mathrm{~m}$ de profondeur, on n'observe aucune teneur significative en or.

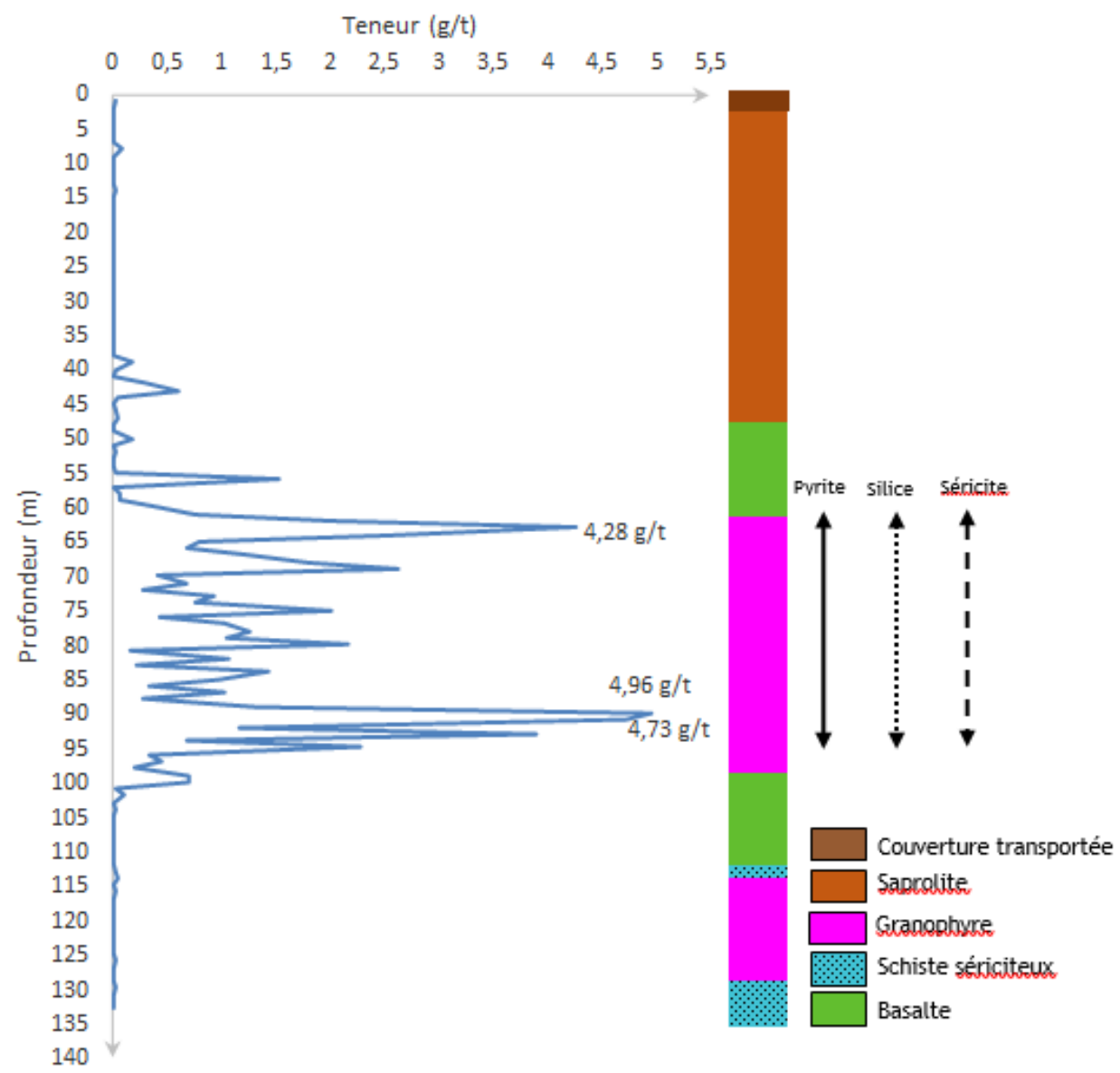

Figure 11 : Evolution de la teneur en or le long du sondage FDC0831.

\section{- $\quad$ Minéralisation filonienne}

La minéralisation filonienne est quelquefois observée entre les plans de schistosité et majoritairement dans les épontes des veines de quartz et dans les veines elles-mêmes. Pour exemple le graphe des teneurs en or du sondage FDE225 (Fig. 12) a montré que les fortes teneurs supérieures à 4 g/t, sont obtenues dans les zones marquées par la présence de veines de quartz dans la couche oxydée entre 15 et $59 \mathrm{~m}$. Entre 110 et $115 \mathrm{~m}$, le pic de teneur à $1,75 \mathrm{~g} / \mathrm{t}$, enregistré dans le basalte est lié à une veine de quartz laiteux 
comportant de la pyrite $(1 \%)$. De même dans le schiste sériciteux, entre 139 et $140 \mathrm{~m}$, la teneur à $2,2 \mathrm{~g} / \mathrm{t}$ d'or, est lié à une veine de quartz.

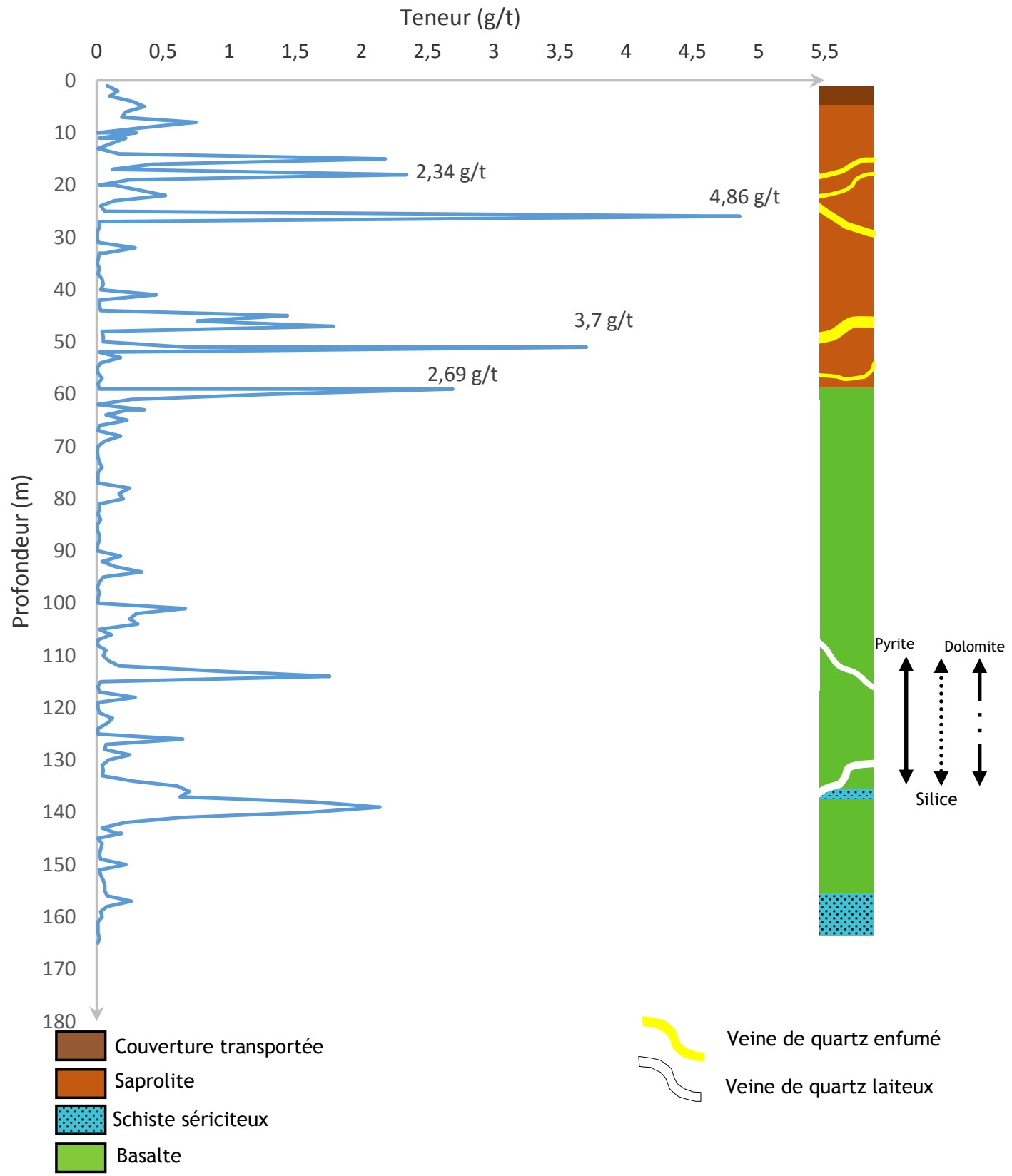

Figure 12 : Evolution de la teneur en or le long du sondage FDE225.

Une minéralisation liée aux veines en feuillet comportant une faible quantité de pyrite ( $1 \%$ ) observé à Dougbafla Nord est également bien mis en exergue par le sondage FDN044 (Fig. 13). Dans ce sondage, la 
minéralisation est présente dans toutes les formations traversées (basalte, microdiorite et granophyre), mais les plus fortes teneurs enregistrées sont observées dans les passages à veines en feuillet $(2,81 \mathrm{~g} / \mathrm{t})$ entre 36 et $40 \mathrm{~m}$ profondeur dans le basalte et $(7 \mathrm{~g} / \mathrm{t})$ entre 95 et $99 \mathrm{~m}$ de profondeur dans le granophyre.

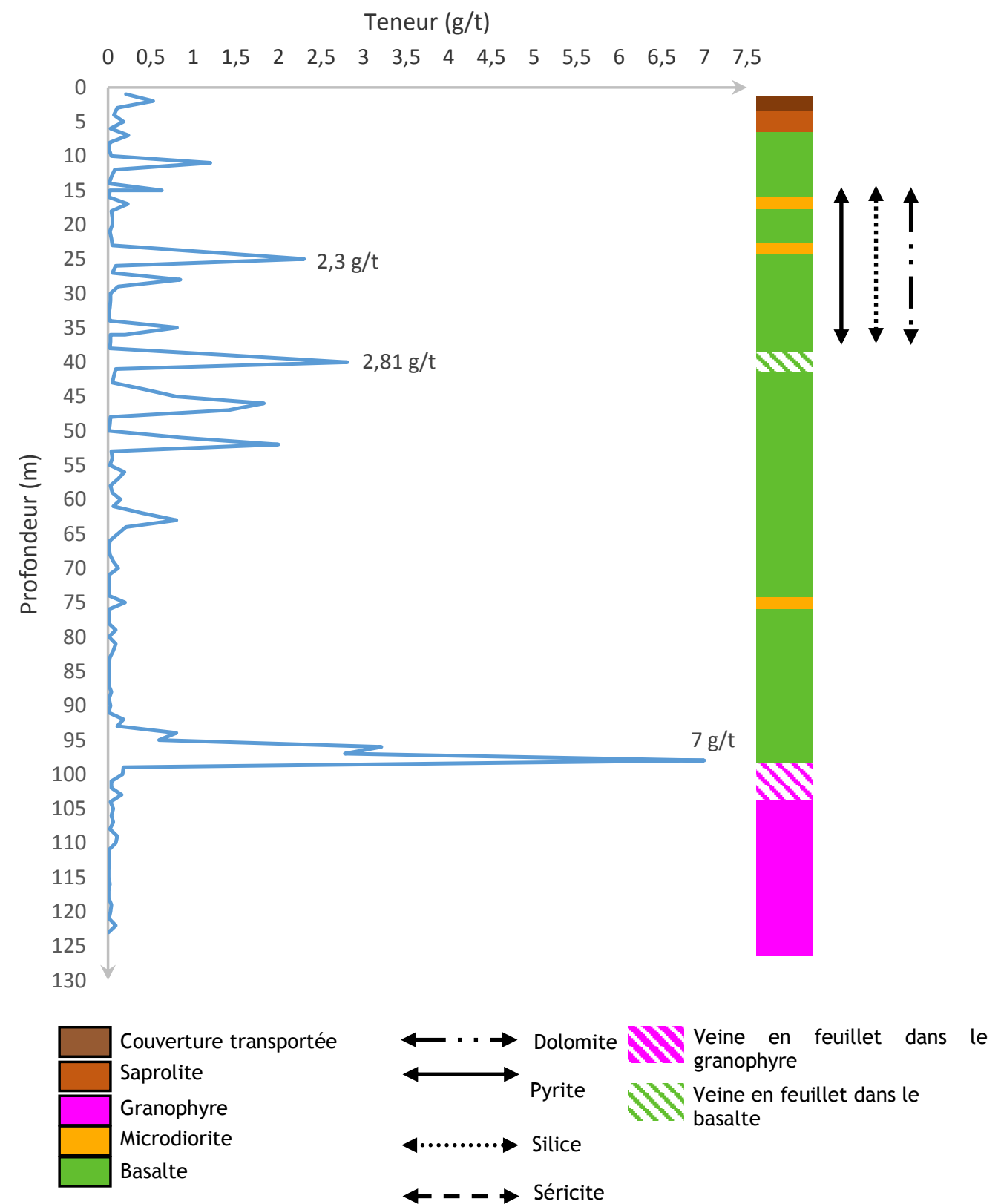

Figure 13 : Evolution de la teneur en or le long du sondage FDN044. 


\section{Discussion}

Dans le gisement de Dougbafla, les zones imprégnées par les fluides hydrothermaux sont matérialisées d'une part, par une forte altération pervasive en séricite, en silice et en carbonate (dolomite) et d'autre part, par une altération filonienne liée à des veines de quartz.

L'analyse des graphes met en évidence l'existence d'une corrélation positive entre les zones à teneurs élevées en or, les zones à altérations pervasives et les zones à sulfures contrairement aux zones saines. De même, les zones pourvues de veines de quartz induites par l'altération filonienne coïncident généralement avec des teneurs élevées en or. Cette corrélation entre l'altération hydrothermale (pervasive et filonienne) et les sulfures permet de définir deux types de minéralisation aurifère. Il s'agit d'une part, d'une minéralisation disséminée liée à des sulfures (pyrite, pyrrhotite, arsénopyrite) et d'autre part, d'une minéralisation filonienne liée aux veines de quartz (planaire ou en feuillet).

La minéralisation à or et sulfures disséminés est intimement liée aux intrusions de granophyre dépourvu de toute veine de quartz. Ce type de minéralisation présente donc une origine magmatique contrôlée par la lithologique dans laquelle l'or se serait mise en place de manière syngénétique. La minéralisation disséminée a également été évoquée dans la plupart des gisements de Côte d'Ivoire : Tongon (Randgold, 2001), Angovia (Coulibaly et al., 2008), Aféma (Assié, 2008 ; Kadio et al., 2010), Agbahou (Houssou, 2013), gîte de Bobosso (Gnanzou, 2014) et Bonikro (Ouattara, 2015).

La minéralisation filonienne représentée par les veines de quartz planaire, irrégulière et en feuillet plus ou moins laiteux ou enfumé, est composée en grande partie de quartz (95\%), de carbonate, de plagioclases et de sulfures (pyrite, pyrrhotite, arsénopyrite) et dans certains cas d'or natif. Cette forme de minéralisation observée principalement dans les zones de cisaillement serait mise en place sous contrôle structural consécutif à l'action de la déformation. La minéralisation filonienne a déjà été évoquée dans la plupart des gisements du Birimien de la Côte d'Ivoire Tongon (Randgold, 2001), Angovia (Coulibaly et al., 2008), Aféma (Assié, 2008 ; Kadio et al., 2010), Agbahou (Houssou, 2013), gîte de Bobosso (Gnanzou, 2014) et Bonikro (Ouattara, 2015), d'Afrique de l'Ouest (Milési et al., 1989 et 1992) et dans la mine sigma au Canada (Robert et al., 1983).

Cette distribution bimodale (disséminée et filonienne) de l'or dans la zone d'étude, a été également mise en évidence dans les gisements de la ceinture d'Ashanti au Ghana (Oberthür et al., 1996), en Côte d'Ivoire dans le gisement d'Agbahou (Houssou, 2013), dans le gîte de Bobosso (Gnanzou, 2014) et dans le gisement de Bonikro (Ouattara, 2015), au Sénégal dans le 
gisement de Massawa (Treloar et al., 2014) et dans d'autres gisements mondialement connus.

\section{Conclusion}

Les zones minéralisées du gisement de Dougbafla encaissées dans les formations volcano-sédimentaires ont montré un lien étroit entre l'altération hydrothermale et les dépôts de sulfures. En fonction de la nature de l'altération hydrothermale, il a été identifié deux modes de concentrations aurifères.

Le premier mode de concentration, appelé minéralisation disséminée, est caractérisé par une distribution diffuse des sulfures (pyrite, pyrrhotite et arsénopyrite) et de l'or dans la matrice des granophyres. Ce type de minéralisation syngénétique est principalement contrôlé par la lithologie (granophyre) dans laquelle l'hydrothermalisme a provoqué une altération de la paragenèse primaire de l'encaissant, soulignée par la silicification, la séricitisation et la dolomitisation. Les chlorites des granophyres, des andésites et des sédiments ont des compositions chimiques de ripidolite. On note accessoirement des chlorites de type pycnochlorite dans les granophyres. L'estimation des températures de cristallisation des chlorites liées à l'épisode hydrothermal, par le géothermomètre "chlorite" a donné des valeurs comprises entre 315 et $328^{\circ} \mathrm{C}$ pour les schistes noirs et les andésites, tandis que celle des granophyres, varie entre 298 et $304^{\circ} \mathrm{C}$.

Le deuxième mode de concentration appelé minéralisation filonienne, est induit par l'altération filonienne caractérisée par des veines de quartz associé à des sulfures (pyrite, pyrrhotite et arsénopyrite). Ce type de minéralisation est contrôlé par la tectonique.

\section{Remerciements}

Les auteurs voudraient témoigner leur reconnaissance à l'endroit de la société Newcrest Mining Ltd qui a financé ce travail ainsi qu'à Laurent MILLO et aux Docteurs Giovanni FUNAIOLI et François LIEBEN pour leurs critiques et suggestions.

\section{References:}

1. Abouchami, W., Boher, M., Michard, A. \& Albarède, F. (1990). A major 2, $1 \mathrm{Ga}$ event of mafic magmatism in West Africa: an early stage of crustal accretion. J. Geophys. Res. 95, pp. 17605-17629.

2. Arnould, G. (1945). Les ressources minières de l'Afrique Occidentale. Bull. Dir. Mines A.O.F., Dakar, $n^{\circ} 8,100 p$.

3. Arnould, A. (1961). Etude géologique des migmatites et des granites précambriens du NE de la Côte d'Ivoire et de la Haute Volta 
méridionale. Thèse Clennont Ferrand. 1960. Mém. BRGM, Fr., $n^{\circ} 3$. $174 p$.

4. Assié, K. E. (2008). Lode gold mineralization in the Paleoproterozoic (Birimian) volcano-sedimentary sequence of Aféma gold district, southeastern Côte d'Ivoire. Thesis, Faculty of Energy and Economic Sciences Technical, University of Clausthal, Germany, 198 p.

5. Bard, J. P. (1974). Remarque à propos de l'évolution géotectonique du craton Ouest-Africain en Côte d'Ivoire. C.R. Acad. Sc. Paris, t. 278, Série D, pp. 2405-2408.

6. Bayliss, P. (1975). Nomenclature of the trioctahedral chlorites. Canadian Mineralogist, vol. 13, pp. 178-180.

7. Boher, M., Abouchami, W., Michard, A., Albarède, F. \& Arndt, N. T. (1992). Crustal growth in West Africa at 2.1 Ga. J. Geophys. Res. Vol. 97, pp. 345-369.

8. Bonhomme, M. (1962). Contribution à l'étude géochronologique de la plate- forme de l'Ouest africain. Ann. Fac. Sc., Univ. ClermontFerrand, $n^{\circ} 5,62 p$.

9. Cathelineau, M. \& Nieva, D. (1985). A chlorite solid solution geothermometer. The Los Azufres (Mexico) geothermal system. Contrib. Mineral Petrol, 91, pp. 235-244.

10. Coulibaly, Y., Kouamelan, A. N., Djro, S. C., Pothin, K. B. K. \& Boffoué, M. O. (2008). Les altérations associées à la minéralisation aurifère d'Angovia (massif du Yaouré, Centre de la Côte d'Ivoire). Revue Ivoirienne des sciences et Technologies $n^{\circ} 11$, pp. 159-175.

11. Daouda, Y. B. (1998). Lithostratigraphie et pétrologie des formations birimiennes de Toumodi-Fettêkro, Côte d'Ivoire : implication pour l'évolution crustale du Paléo-protérozoïque du craton Ouest-Africain. Doctorat, Univ. Orléans-Géosciences, 737, 191 p.

12. Doumbia, S., Pouclet, A., Kouamelan, A. N., Peucat, J. J., Vidal, M. \& Delor, C. (1998). Petrogenesis of juvenile-type Birimian (Paeoproterozoic) granitoids in Central Côte d'Ivoire, West Africa: geochemistry and geochronology. Precambrian Res., 87, pp. 33-63.

13. Deer, W. A., Howie, R. A. \& Zussman, J. (1992). An introduction to the rock forming minerals. $2^{\text {nd }} e d$., Pearson Education Limited, Harlow, 696 .

14. Equigold, CI SA (2007). Rapport d'exploration du permis d'Oumé (PR105), $11 p$.

15. Feybesse, J. L., Milesi, J. P., Johan, Y., Dommanget, A., Calvez, J. Y., Boher, M. \& Abouchamy, W. (1989). La limite Archéen Protérozoïque d'Afrique de l'Ouest : une zone de chevauchement antérieure à l'accident de Sassandra ; l'exemple des régions 
d'Odienné et de Touba (Côte d'Ivoire). C. R. Acad. Sci. Paris, 309, pp. 1847-1853.

16. Geomines Ltd (1982). Inventaire hydrogéologique appliqué à l'hydraulique villageoise. Ministère des Travaux Publics et des Transports, Direction Centrale de l'Hydraulique, République de Côte d'Ivoire, carte de Gagnoa, Cahier n ${ }^{\circ} 24,31$.

17. Gnanzou, A. (2014). Etude des séries volcanosédimentaires de la région de Dabakala (Nord-Est de la Côte d'Ivoire): Genèse et évolution magmatique. Contribution à la connaissance de la minéralisation aurifère de Bobosso dans la série de la Haute-Comoé. Doctorat, Univ. PARIS-SUD XI, France et Univ. F. H. B, Abidjan, $303 \mathrm{p}$.

18. Houssou, N. N. (2013). Etude pétrographique, structurale et métallogénique du gisement aurifère d'Agbahou, Divo, Côte d'Ivoire. Doctorat, Univ. Felix HOUPHOUET-BOIGNY, 177 p.

19. Marcelin, J. (1971). Notice explicative de la carte géologique à $1 / 200$ 000 Goua-Batié. Dir. Mines Haute-volta, 31 p.

20. Mortimer, J. (1992). Lithostratigraphy of the early Proterozoic Toumodi volcanic Group in Central Côte d'Ivoire: implications for Birrimian stratigraphic models. Journal of African Earth Sciences, 14, 1, pp. 81-91.

21. Kadio, E., Coulibaly, Y., Allialy, M. E., Kouamelan, A.N., \& Pothin, K.B.K. (2010). On the occurrence of gold mineralizations in southeastern Ivory Coast. Journal of African Earth Sci., 57, pp. 423430.

22. Milesi, J. P., Feybesse, J. L., Ledru, P., Dommanget, A., Ouedraogo, M. F., Marcoux, E., Prost, A., Vinchon, C., Sylvain, J. P., Johan, V., Tegyey, M., Calvez, J. Y. \& Lagny, P. (1989). Les minéralisations aurifères de l'Afrique de l'Ouest, leurs relations avec l'évolution lithostructurale du Protérozoïque inférieur. Chron. Rech. Min., Fr., 497, $98 p$.

23. Oberthür, T., Schmidt, M. A., Vetter, U., Simon, K. \& Amanor, J. A. (1996). Gold mineralization in the Ashanti belt of Ghana: genetic constraints of the stable isotope geochemistry. Econ. Geol., (91), 2, pp. 289-301.

24. Ouattara, Z. (2015). Caractères lithostratigraphiqie, structural, géochimique et métallogénique du gisement d'or de Bonikro, sillon birimien de Fettekro, centre-sud de la Côte d'Ivoire. Doctorat, Univ. Felix HOUPHOUET-BOIGNY, 256 p.

25. Randgold Resources Ltd (2001). Permis de Niellé. Rapport final, 90 p. 
26. Roques, M. (1948). Le Précambrien de l'Afrique Occidentale française.Bull. Soc. Géol. Fr., $5^{\circ}$ Série, t. XVIII, fasc. 8-9. Paris. pp. 589-628.

27. Sonnendrucker, P. (1969). Etude de synthèse sur l'or en Côte d'Ivoire. Rapport de fin de mission, SODEMI, Abidjan, rapport $n^{\circ} 222,127$ p. multigr.

28. Soule de Lafont, D. (1956). Le Précambrien moyen et supérieur de Bondoukou (Côte d'Ivoire). Bull. Dir. Féd. Mines-Géol. Dakar, $N^{\circ} 18,163 p$.

29. Tagini, B. (1971). Esquisse structurale de la Côte d'Ivoire. Essai géotechnique régional, Doctorat Univ. Fac. Des Sci. Lausanne (Suisse), et, SODEMI, Abidjan, 302 p.

30. Yacé, I. (1984). Le Précambrien de l'Afrique de l'Ouest et ses corrélations avec le Brésil oriental. Rapport final. Publication PICGCIFEG, $N^{\circ} 108-144$, Paris, $28 p$.

31. Tempier, P. (1986). Le Burkinien: cycle orogénique majeur du protérozoïque inférieur en Afrique de l'Ouest. Publi. Occas. CIFEG, $n^{\circ} 10, p p .17-23$.

32. Trdlicka, Z. \& Hoffman, V. (1976). Untersuchungen der chemischen Zusammensetzung der Gangkarbonate von Kutna Hora / CSSR. Freiberger Forschungshefte, vol. C, 321, pp. 29-81.

33. Treloar, P. J., Lawrence, D. M., Senghor, D., Boyce, A. \& Harbidge, P. (2014). The Massawa gold deposit, Eastern Senegal, West Africa: an orogenic gold deposit sourced from magmatically derived fluids? Geological Society, London, special publications, doi.1144/SP393, $12,27 \mathrm{p}$. 